\title{
Depth-Assisted Rectification of Patches Using RGB-D Consumer Devices to Improve Real-Time Keypoint Matching
}

\author{
João Paulo Lima ${ }^{1}$, Francisco Simões ${ }^{1}$, Hideaki Uchiyama ${ }^{2}$, Veronica Teichrieb ${ }^{1}$ and Eric Marchand ${ }^{2}$ \\ ${ }^{I}$ Voxar Labs, Informatics Center, Federal University of Pernambuco, Av. Jorn. Anibal Fernandes, S/N, Recife, Brazil \\ ${ }^{2}$ INRIA Rennes Bretagne-Atlantique, Rennes, France \\ \{jpsml, fpms, vt\}@cin.ufpe.br, \{Hideaki.Uchiyama, Eric.Marchand\}@ inria.fr
}

\begin{abstract}
Keywords: Keypoint Matching, Pose Estimation, Computer Vision, RGB-D.
Abstract: $\quad$ This paper presents a method named Depth-Assisted Rectification of Patches (DARP), which exploits depth information available in RGB-D consumer devices to improve keypoint matching of perspectively distorted images. This is achieved by generating a projective rectification of a patch around the keypoint, which is normalized with respect to perspective distortions and scale. The DARP method runs in real-time and can be used with any local feature detector and descriptor. Evaluations with planar and non-planar scenes show that DARP can obtain better results than existing keypoint matching approaches in oblique poses.
\end{abstract}

\section{INTRODUCTION}

This paper introduces a novel technique for object detection and tracking named Depth-Assisted Rectification of Patches (DARP). The proposed technique can provide rotation, scale and perspective invariant features based on a patch rectification approach. The DARP method is designed to make use of RGB-D sensors, such as consumer devices like Microsoft Kinect, and exploit both image and depth data to improve feature detection and description. RGB-D sensors became in the last years a low cost consumer product of easy access to general users. The DARP technique uses the depth data provided by such sensors to estimate normals on the scene surface of $3 \mathrm{D}$ points that correspond to keypoints extracted from the RGB image. The depth data is also used together with the estimated normals to rectify patches around the keypoints with fixed size in camera coordinates. This contributes to remove perspective distortions caused by oblique poses and scale changes, and it shall be demonstrated that using the descriptors computed from the rectified patches for real-time keypoint matching can give improved results.

Since perspective deformations can be approximated by affine transformations for small areas, affine invariant local features can be used to generate normalized patches (Mikolajczyk et al., 2005). On the other hand, DARP can use local features that are, a priori, not affine and scale invariant, performing a posteriori projective rectification of the patches. The ASIFT method (Morel and $\mathrm{Yu}, 2009$ ) obtains a higher number of matches from perspectively distorted images by generating several affine transformed versions of both images and then finding correspondences between them using SIFT. Alternatively, the DARP method is able to use solely the query and template images in order to match them. ASIFT also makes use of low-resolution versions of the affine transformed images in order to accelerate keypoint matching. Only the affine transforms that provide more matches are used to compare the images in their original resolution. The DARP technique is able to work directly with high resolution images, without needing to decrease their quality to achieve real-time keypoint matching. In (Koser and Koch, 2007), MSER features are projectively rectified using Principal Component Analysis (PCA) and graphics hardware. However, it does not focus on real-time execution and it is designed to work with region detectors, while the DARP method works with keypoint detectors and computes rectified patches in real-time. Patch perspective rectification is also performed in (Del Bimbo et al., 2010), (Hinterstoisser et al., 2008), (Hinterstoisser et al., 2009) and (Pagani and Stricker, 2009). These methods differ from DARP because they first estimate patch identity and coarse pose, and then refine the pose of the identified patch. In DARP, the patches are first rectified in order to allow estimating their identity. In addition, these methods need to previously generate warped versions of the patch for 
being able to compute its rectification, while DARP can rectify a patch without such constraint. The methods described in (Eyjolfsdottir and Turk, 2011), (Kurz and Benhimane, 2011), (Wu et al., 2008) and (Yang et al., 2010) first projectively rectify the whole image and then detect invariant features on the normalized result, while the DARP method does the opposite. In addition, ( $\mathrm{Wu}$ et al., 2008) is designed for offline 3D reconstruction, (Eyjolfsdottir and Turk, 2011), (Kurz and Benhimane, 2011) and (Yang et al., 2010) target only planar scenes and (Eyjolfsdottir and Turk, 2011) and (Kurz and Benhimane, 2011) require an inertial sensor. Concurrent with this research (Marcon et al., 2012) used an RGB-D sensor to perform patch rectification using PCA, followed by 2D Fourier-Mellin Transform for description. Nevertheless, the rectification algorithm applied is not clearly described, it is not evaluated under a real-time keypoint matching scenario and only planar scenes are used in the experiments.

The contributions of this paper are: (1) patch rectification method that uses depth information to obtain a perspective and scale invariant representation of keypoints; (2) qualitative and quantitative evaluation of the technique, showing that it is suitable to both planar and non-planar scenes and provides good results in oblique poses; (3) runtime analysis of the method, which shows that it runs in real-time.

This paper is organized as follows. Section 2 describes each step of the DARP technique. Section 3 discusses the results obtained with DARP and presents qualitative and quantitative evaluations with respect to keypoint matching quality and runtime performance. Conclusions and future work are detailed in Section 4.

\section{DEPTH-ASSISTED RECTIFICATION OF PATCHES}

In DARP, keypoints are extracted and their normal vectors on the scene surface are estimated using the depth image. Then, using depth and normal information, patches around the keypoints are rectified to a canonical view in order to remove perspective and scale distortions. For rotation invariance, the rectified patch orientation is computed using geometric moments. A descriptor for the rectified patch is computed using the assigned orientation. The query descriptors are matched with previously obtained template descriptors. Camera pose can then be estimated from the correspondences. Each step of the DARP method is detailed on the next subsections.

\subsection{Keypoint Detection}

Any keypoint detector can be used by DARP. Since the patch around the keypoint is normalized a posteriori with respect to perspective distortions and scale, the detector does not have to be affine or scale invariant and the use of a scale pyramid for the input image is not mandatory. In the current implementation, the DARP method uses FAST-9 (Rosten and Drummond, 2006), since it presents a good tradeoff between efficiency and quality. The keypoints are detected using the original scale of the input image, without employing a scale pyramid. An initial set of features is detected on the input image and then $n$ points with best Harris response are selected. It was used a value of $n=230$ in the conducted experiments.

\subsection{Normal Estimation}

From the query depth image, a 3D point cloud in camera coordinates can be computed for the scene. Considering a 3D point $\boldsymbol{M}_{\text {cam }}=\left[M_{x}, M_{y}, M_{z}\right]^{T}$ in camera coordinates, its $2 \mathrm{D}$ projection $\boldsymbol{m}=$ $\left[m_{x}, m_{y}, 1\right]^{T}$ is given by:

$$
\begin{gathered}
\boldsymbol{m}=\left[\begin{array}{c}
f_{x} M_{x} / M_{z}+c_{x} \\
f_{y} M_{y} / M_{z}+c_{y}
\end{array}\right]= \\
\underbrace{\left[\begin{array}{ccc}
f_{x} & 0 & c_{x} \\
0 & f_{y} & c_{y} \\
0 & 0 & 1
\end{array}\right]}_{K}\left[\begin{array}{c}
M_{x} / M_{z} \\
M_{y} / M_{z} \\
1
\end{array}\right],
\end{gathered}
$$

where $f_{x}$ and $f_{y}$ are the focal length in terms of pixel dimensions in the $x$ and $y$ direction respectively, $c_{x}$ and $c_{y}$ are the coordinates of the principal point and $K$ is known as the intrinsic parameters matrix. Thus, rearranging the terms and considering $M_{z}=d$, where $d$ is the depth of $\boldsymbol{m}$, the coordinates of $\boldsymbol{M}_{\boldsymbol{c a m}}$ can be obtained by:

$$
\boldsymbol{M}_{\text {cam }}=\left[\begin{array}{c}
\left(m_{x}-c_{x}\right) \cdot d / f_{x} \\
\left(m_{y}-c_{y}\right) \cdot d / f_{y} \\
d
\end{array}\right]
$$

Using this point cloud, a normal vector can be estimated for a 3D point $\boldsymbol{M}_{\boldsymbol{c a m}}$ that corresponds to an extracted 2D keypoint via PCA. The centroid $\overline{\boldsymbol{M}}$ of all neighbour 3D points $\boldsymbol{M}_{\boldsymbol{i}}$ within a radius of $3 \mathrm{~cm}$ of $\boldsymbol{M}_{\text {cam }}$ is computed. A covariance matrix is 
computed using $\boldsymbol{M}_{\boldsymbol{i}}$ and $\overline{\boldsymbol{M}}$, and its eigenvectors $\left\{\boldsymbol{v}_{1}, \boldsymbol{v}_{2}, \boldsymbol{v}_{3}\right\}$ and corresponding eigenvalues $\left\{\lambda_{1}, \lambda_{2}, \lambda_{3}\right\}$ are computed and ordered in ascending order. The normal vector to the scene surface at $\boldsymbol{M}_{\boldsymbol{c a m}}$ is given by $\boldsymbol{v}_{\mathbf{1}}$ (Berkmann and Caelli, 1994). If needed, $\boldsymbol{v}_{\mathbf{1}}$ is flipped to point towards the viewing direction. Only the keypoints that have a valid normal are kept.

\subsection{Patch Rectification}

The next step consists in using the available 3D information to rectify a patch around each keypoint in order to remove perspective deformations. In addition, a scale normalized representation of the patch is obtained. This is done by computing a homography that transfers the patch to a canonical view, as illustrated in Figure 1. Given $\boldsymbol{n}=$ $\left(n_{x}, n_{y}, n_{z}\right)^{T}$ as the unit normal vector in camera coordinates at $\boldsymbol{M}_{\text {cam }}$, which is the corresponding 3D point of a keypoint, two unit vectors $\boldsymbol{n}_{\mathbf{1}}$ and $\boldsymbol{n}_{\mathbf{2}}$ that define a plane with normal $\boldsymbol{n}$ can be obtained by:

$$
\begin{gathered}
\boldsymbol{n}_{\mathbf{1}}=\frac{1}{\left\|\left(n_{z}, 0,-n_{x}\right)^{T}\right\|} \cdot\left(n_{z}, 0,-n_{x}\right)^{T}, \\
\boldsymbol{n}_{\mathbf{2}}=\boldsymbol{n} \times \boldsymbol{n}_{\mathbf{1}} .
\end{gathered}
$$

This is valid because it is assumed that $n_{x}$ and $n_{z}$ are not equal to zero at the same time, since in this case the normal would be perpendicular to the viewing direction and the patch would be not visible.

From $\boldsymbol{M}_{\text {cam }}, \boldsymbol{n}_{\mathbf{1}}$ and $\boldsymbol{n}_{\mathbf{2}}$, it is possible to find the 3D corners $\boldsymbol{M}_{\mathbf{1}}, \ldots, \boldsymbol{M}_{\mathbf{4}}$ of the patch in the camera coordinate system. The patch size in camera coordinates should be fixed in order to allow scale invariance. The $2 \mathrm{D}$ corners $\boldsymbol{m}_{\mathbf{1}}, \ldots, \boldsymbol{m}_{\mathbf{4}}$ of the patch to be rectified in image coordinates are the projection of the $3 \mathrm{D}$ points $\boldsymbol{M}_{\mathbf{1}}, \ldots, \boldsymbol{M}_{\mathbf{4}}$, i.e., $\boldsymbol{m}_{\boldsymbol{i}}=K \boldsymbol{M}_{\boldsymbol{i}}$. If the patch size in image coordinates is too small, the rectified patch will suffer degradation in image resolution, harming its description. This size is influenced by the location of the $3 \mathrm{D}$ point $\boldsymbol{M}_{\boldsymbol{c} \boldsymbol{c} \text { m }}$ (e.g., if $\boldsymbol{M}_{\boldsymbol{c a m}}$ is too far from the camera, the patch size will be small). It is also directly proportional to the patch size in camera coordinates, which is determined by a constant factor $k$ applied to $\boldsymbol{n}_{\mathbf{1}}$ and $\boldsymbol{n}_{\mathbf{2}}$ as follows: $\boldsymbol{n}_{\mathbf{1}}{ }^{\prime}=k \cdot \boldsymbol{n}_{\mathbf{1}}$ and $\boldsymbol{n}_{\mathbf{2}}{ }^{\prime}=k \cdot \boldsymbol{n}_{\mathbf{2}}$. The factor $k$ should be large enough to allow good scale invariance while being small enough to give distinctiveness to the patch. In the performed experiments, it was used $k=\lfloor s / 2\rfloor$, where $s$ is the size of the rectified patch (set to 31 in the tests).

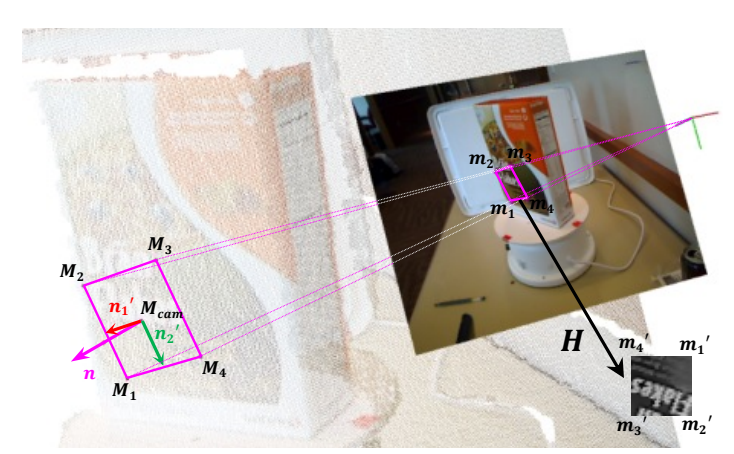

Figure 1: Patch rectification overview. $\boldsymbol{M}_{\mathbf{1}}, \ldots, \boldsymbol{M}_{\mathbf{4}}$ are computed from $\boldsymbol{M}_{\boldsymbol{c a m}}, \boldsymbol{n}_{\mathbf{1}}{ }^{\prime}$ and $\boldsymbol{n}_{\mathbf{2}}{ }^{\prime}$. An homography $\boldsymbol{H}$ is computed from the projections $\boldsymbol{m}_{\mathbf{1}}, \ldots, \boldsymbol{m}_{\mathbf{4}}$ and the canonical corners $\boldsymbol{m}_{\mathbf{1}}{ }^{\prime}, \ldots, \boldsymbol{m}_{\mathbf{4}}{ }^{\prime}$. by:

The corners $\boldsymbol{M}_{\mathbf{1}}, \ldots, \boldsymbol{M}_{\mathbf{4}}$ of the patch are given

$$
\begin{aligned}
& M_{1}=M_{c a m}+n_{1}{ }^{\prime}+n_{2}{ }^{\prime} \\
& M_{2}=M_{c a m}+n_{1}{ }^{\prime}-n_{2}{ }^{\prime} \\
& M_{3}=M_{c a m}-n_{1}{ }^{\prime}-n_{2}{ }^{\prime} \\
& M_{4}=M_{c a m}-n_{1}{ }^{\prime}+n_{2}{ }^{\prime}
\end{aligned}
$$

The corresponding corners $\boldsymbol{m}_{\mathbf{1}}{ }^{\prime}, \ldots, \boldsymbol{m}_{\mathbf{4}}{ }^{\prime}$ of the patch in the canonical view are:

$$
\begin{aligned}
& \boldsymbol{m}_{\mathbf{1}}{ }^{\prime}=(s-1,0)^{T}, \\
& \boldsymbol{m}_{\mathbf{2}}{ }^{\prime}=(s-1, s-1)^{T}, \\
& \boldsymbol{m}_{\mathbf{3}}{ }^{\prime}=(0, s-1)^{T}, \\
& \boldsymbol{m}_{\mathbf{4}}{ }^{\prime}=(0,0)^{T} .
\end{aligned}
$$

From $\boldsymbol{m}_{1}, \ldots, \boldsymbol{m}_{\mathbf{4}}$ and $\boldsymbol{m}_{\mathbf{1}}{ }^{\prime}, \ldots, \boldsymbol{m}_{\mathbf{4}}{ }^{\prime}$, it can be computed an homography $\boldsymbol{H}$ that takes points of the input image to points of the rectified patch.

\subsection{Orientation Estimation}

In order to achieve rotational invariance, patch orientation can be estimated based on the intensity centroid, which is computed from geometric moments (Rublee et al., 2011). Compared to gradient-based methods, the use of intensity centroid has the advantage of presenting a uniformly good orientation even under large image noise (Rublee et al., 2011), besides being fast to compute.

\subsection{Patch Description}

The same way DARP can use any keypoint detector, it is also possible to have any patch descriptor. In the current implementation, the Rotation-Aware BRIEF (rBRIEF) was used due to its good performance and simple rotational invariance treatment (Rublee et al., 2011). The rBRIEF descriptor is based on binary 
tests over a smoothed patch, but with steered versions of the tests. Each steered pattern corresponds to a discretized rotation angle applied to the coordinates of the binary tests. A lookup table of the steered patterns is created to speed up the matching process. In addition, a learning approach is used in order to obtain a good set of binary tests. At runtime, the steered version to be used for generating the descriptor is chosen based on the assigned orientation of the patch. DARP used 30 distinct angles for the discretization.

\subsection{Descriptor Matching and Pose Estimation}

For descriptor matching, a nearest neighbour search is performed in order to find the corresponding template descriptor for each query descriptor. In the tests performed, a brute force search with Hamming distance was applied, where matches with a distance greater than 50 are discarded.

Regarding pose estimation, if the objects present on the scene are planar, homography estimation can be used to compute their pose. If the scene is nonplanar, a Perspective- $n$-Point $(\mathrm{P} n \mathrm{P})$ method can be applied. In the experiments performed, planar objects pose were computed using homography estimation with the DLT method, while non-planar objects pose were estimated using the EP $n \mathrm{P}$ method (Moreno-Noguer et al., 2007). In both cases, the RANSAC algorithm was applied for outliers removal.

\section{RESULTS}

In order to evaluate DARP, some image sequences from the publicly available University of Washington's RGB-D Object Dataset (Lai et al., 2011) were used and synthetic RGB-D images were also generated. All the experiments were performed with $640 \times 480$ images. The hardware used in the evaluation was a Microsoft Kinect for Xbox 360 and a laptop with Intel Core i7-3612QM @ 2.10GHz processor and 8GB RAM.

The results obtained with DARP were compared with ORB (Rublee et al., 2011), since the current implementation of DARP performs keypoint detection, orientation assignment and patch description in a way similar to ORB. It should be noted that ORB uses an image pyramid with 5 levels and a scale factor of 1.2 in order to obtain scale invariance, while DARP does not make use of an image pyramid, since scale changes are inherently handled using the patch rectification process. ORB extracts 631 keypoints per image pyramid, distributed in the levels in ascending order as follows: 230, 160, 111, 77 and 53 keypoints. The DARP technique uses only the 230 keypoints extracted from the first level. Two images of the same object with different poses were matched using both techniques. Descriptor matching and pose estimation were performed using the same procedures for both DARP and ORB, as described in Subsection 2.6.

\subsection{Qualitative Evaluation}

Initially the tests were done with planar objects. It is shown in Figure 2 and Figure 4 the matches between the two instances of a planar object in the frames cereal box_1_1_176 and cereal_box_1_1_208 from the University of Washington's RGB-D Object Dataset. The 2D points that belong to the object model transformed by the homographies computed from the matches are shown in Figure 3 and Figure 5. It can be noted that the DARP method provides better results than ORB when the object has an oblique pose with respect to the viewing direction. The matches obtained with ORB led to a wrong pose, while it was possible to estimate a reasonable pose using DARP, as evidenced by the transformed model points.

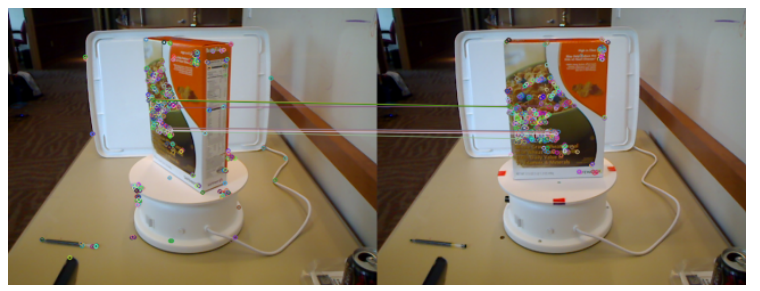

Figure 2: Planar object keypoint matching using ORB finds 10 matches.

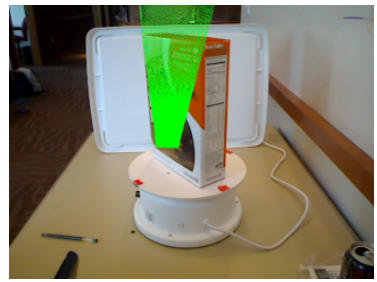

Figure 3: Planar object pose estimation using ORB. 


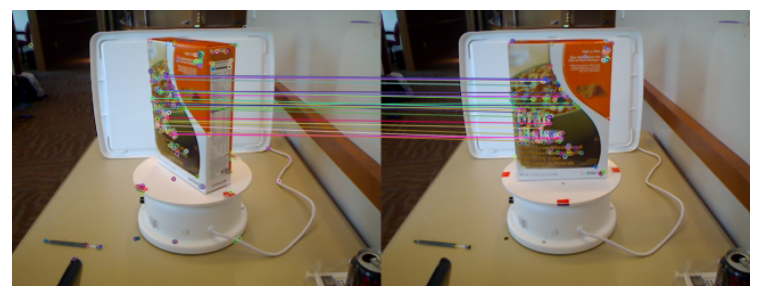

Figure 4: Planar object keypoint matching using DARP finds 27 matches.

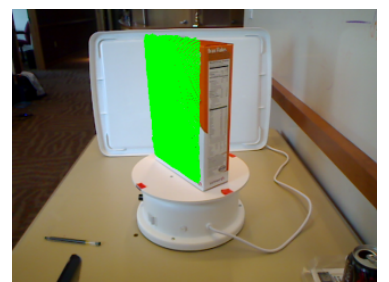

Figure 5: Planar object pose estimation using DARP.

After, some tests were done with non-planar objects. In this case, Figure 8 illustrates the projection of a 3D point cloud model of the object using the pose computed from the matches found by DARP shown in Figure 7 . The frames from the University of Washington's RGB-D Object Dataset used in this experiment are food can $14 \quad 1 \quad 181$ and food can 141 197. DARP also obtained better results than ORB in the oblique pose scenario, since DARP provided matches that allowed computing the object pose, while ORB did not find any valid matches, as can be seen in Figure 6 .

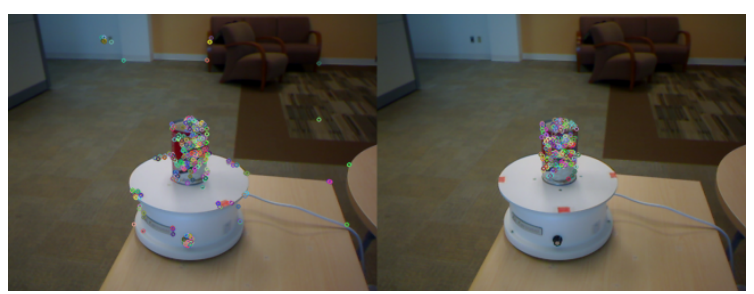

Figure 6: Non-planar object keypoint matching using ORB finds 0 matches.

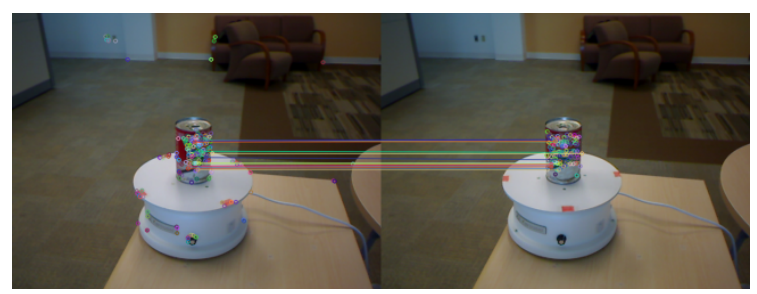

Figure 7: Non-planar object keypoint matching using DARP finds 14 matches.

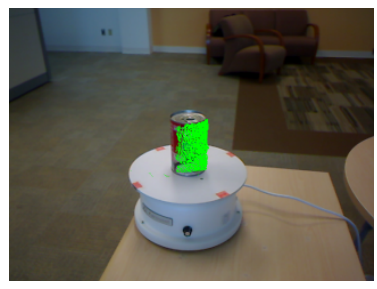

Figure 8: Non-planar object pose estimation using DARP.

\subsection{Quantitative Evaluation}

Keypoint matching quality was evaluated by measuring the correctness of the poses estimated from the matches with a database of 280 synthetic RGB-D images of a planar object (a cereal box) under different viewpoints on a cluttered background. The poses were under a degree change range of $\left[0^{\circ}, 70^{\circ}\right]$ with a $10^{\circ}$ step and a scale range of $[1.0,2.0]$ with a 0.2 step. The percentage of correct poses estimated by each method was calculated. The pose was considered as correct only if the root-mean-square (RMS) reprojection error was below 3 pixels. Figure 9 shows that DARP outperformed ORB in larger viewpoint changes.

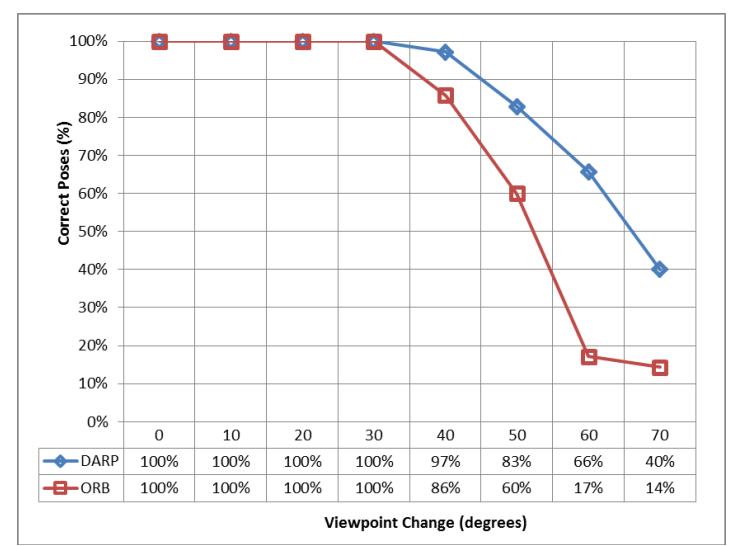

Figure 9: Percentage of correct poses of ORB and DARP with respect to viewpoint change.

\subsection{Performance Analysis}

Table 1 presents the average time and the percentage of time required by each step of ORB and a nonoptimized implementation of the DARP method. It shows that the DARP method runs at $\sim 36 \mathrm{fps}$ and its most time demanding step is the normal estimation phase, which takes more than $50 \%$ of all processing time. The patch rectification step also heavily contributes to the final processing time. ORB takes more time than DARP for keypoint detection and patch description, since it uses an image pyramid and extracts a higher number of keypoints. ORB 
estimates patch orientation in a faster manner than DARP because it makes use of integral images in this step. DARP could be optimized to perform orientation estimation in the same way, but it would not represent a significant performance gain, as this step takes less than $1 \%$ of total processing time.

Table 1: Average computation time and percentage for each step of ORB and DARP methods.

\begin{tabular}{|c|c|c|r|r|}
\cline { 2 - 5 } \multicolumn{1}{c|}{} & \multicolumn{2}{c|}{ ORB } & \multicolumn{2}{c|}{ DARP } \\
\cline { 2 - 5 } \multicolumn{1}{c|}{} & ms & \multicolumn{1}{c|}{$\%$} & \multicolumn{1}{c|}{ ms } & \multicolumn{1}{c|}{$\%$} \\
\hline Keypoint detection & 16.11 & 80.89 & 2.63 & 9.40 \\
\hline Normal estimation & - & - & 14.99 & 53.56 \\
\hline Patch rectification & - & - & 8.40 & 30.01 \\
\hline Orientation estimation & 0.14 & 0.71 & 0.20 & 0.72 \\
\hline Patch description & 3.67 & 18.40 & 1.77 & 6.31 \\
\hline Total & $\mathbf{1 9 . 9 2}$ & $\mathbf{1 0 0 . 0 0}$ & $\mathbf{2 7 . 9 9}$ & $\mathbf{1 0 0 . 0 0}$ \\
\hline
\end{tabular}

\section{CONCLUSIONS}

The DARP method has been introduced, which exploits depth information to improve keypoint matching. This is done by rectifying the patches using the 3D information in order to remove perspective distortions. The depth information is also used to obtain a scale invariant representation of the patches. It was shown that DARP can be used together with existing keypoint matching methods in order to help them to handle situations such as oblique poses with respect to the viewing direction. It supports both planar and non-planar objects and is able to run in real-time.

As future work, tests with other keypoint detectors and patch descriptors will be done. Optimizations on normal estimation and patch rectification are also planned, since they showed to be the most time demanding steps of the technique.

\section{REFERENCES}

Berkmann, J., Caelli, T., 1994. Computation of surface geometry and segmentation using covariance techniques. In IEEE Transactions on Pattern Analysis and Machine Intelligence, volume 16, issue 11, pages 1114-1116.

Del Bimbo, A., Franco, F., Pernici, F., 2010. Local homography estimation using keypoint descriptors. In WIAMIS'10, 11th International Workshop on Image Analysis for Multimedia Interactive Services, 4 pages.

Eyjolfsdottir, E., Turk., M., 2011. Multisensory embedded pose estimation. In WACV'11, IEEE Workshop on Applications of Computer Vision, pages 23-30.
Hinterstoisser, S., Benhimane, S., Navab, N., Fua, P., Lepetit, V., 2008. Online learning of patch perspective rectification for efficient object detection. In CVPR'08, 21th IEEE Conference on Computer Vision and Pattern Recognition, 8 pages.

Hinterstoisser, S., Kutter, O., Navab, N., Fua, P., Lepetit, V., 2009. Real-time learning of accurate patch rectification. In CVPR'09, 22th IEEE Conference on Computer Vision and Pattern Recognition, pages 2945-2952.

Koser, K., Koch, R., 2007. Perspectively invariant normal features. In ICCV'07, 11th IEEE International Conference on Computer Vision, 8 pages.

Kurz, D., Benhimane, S., 2011. Gravity-aware handheld augmented reality. In ISMAR'11, 10th IEEE International Symposium on Mixed and Augmented Reality, pages 111-120.

Lai, K., Bo, L., Ren, X., Fox, D., 2011. A large-scale hierarchical multi-view RGB-D object dataset. In ICRA'11, IEEE International Conference on Robotics and Automation, pages 1817-1824.

Marcon, M., Frigerio, E., Sarti, A., Tubaro, S., 2012. 3D wide baseline correspondences using depth-maps. In Signal Processing: Image Communication, volume 27, issue 8 , pages 849-855.

Mikolajczyk, K., Tuytelaars, T., Schmid, C., Zisserman, A., Matas, J., Schaffalitzky, F., Kadir, T., Van Gool, L., 2005. A comparison of affine region detectors. In International Journal of Computer Vision, volume 5, issue 1-2, pages 43-72.

Morel, J., Yu, G., 2009. ASIFT: A new framework for fully affine invariant image comparison. In SIAM Journal on Imaging Sciences, volume 2, issue 2, pages 438-469.

Moreno-Noguer, F., Lepetit, V., Fua, P., 2007. Accurate non-iterative $\mathrm{O}(\mathrm{n})$ solution to the PnP problem. In ICCV'07, 11th IEEE International Conference on Computer Vision, 8 pages.

Pagani, A., Stricker, D., 2009. Learning local patch orientation with a cascade of sparse regressors. In BMVC'09, 20th British Machine Vision Conference, pages 86.1-86.11.

Rosten, E., Drummond, T., 2006. Machine learning for high-speed corner detection. In ECCV'06, 9th European Conference on Computer Vision, pages 430-443.

Rublee, E., Rabaud, V., Konolige, K., Bradski, G., 2011. ORB: an efficient alternative to SIFT or SURF. In ICCV'11, 15th IEEE International Conference on Computer Vision, pages 2564-2571.

Wu, C., Clipp, B., Li, X., Frahm, J.-M., Pollefeys, M., 2008. 3D model matching with viewpoint invariant patches (VIPs). In CVPR'08, IEEE Conference on Computer Vision and Pattern Recognition, 8 pages.

Yang, M., Cao, Y., Förstner, W., McDonald, J., 2010. Robust wide baseline scene alignment based on $3 \mathrm{~d}$ viewpoint normalization. In ISVC'10, 6th International Symposium on Visual Computing, Lecture Notes in Computer Science, volume 6453, pages 654-665. 